\title{
Les Services Financiers au Maroc - Une Industrie Confrontée à des Risques Excessifs
}

\author{
Jamal Abnaha, Fadwa Errami \\ Université Mohamed 5, Rabat, Maroc
}

\begin{abstract}
Résumé : Les services financiers (FS) sont un secteur très important pour Oxial. Certains de nos premiers clients étaient des sociétés de services financiers, nous travaillons actuellement avec des sociétés de services financiers dans le monde entier et c'est un secteur dans lequel nous avons une grande expérience et des connaissances approfondies. Les services financiers sont devenus plus fortement réglementés que jamais, à la fois au niveau local et mondial, et le secteur est également confronté à plus de risques qu'auparavant. Les services financiers sont plus réglementés que jamais, tant au niveau local que mondial, et le secteur est confronté à des risques plus importants que par le passé. Cela signifie que les prestataires de services financiers doivent être encore plus vigilants en ce qui concerne la gestion et l'atténuation des risques, et qu'ils doivent mettre en place les outils de GRC appropriés pour les aider dans cette tâche et veiller à ce qu'ils restent conformes. Ils disposent de plus de ressources, de personnel et d'accès aux dernières solutions logicielles de GRC. Le Maroc, premier pays non européen où Oxial a ouvert un bureau, est un pays où les services financiers sont plus prospères que dans la plupart des autres pays d'Afrique, et nous examinons dans quelle mesure le secteur est préparé à gérer et à atténuer les risques.
\end{abstract}

Mots-clés : Finance solidaire, relation de service, proximité, développement local.

\section{INTRODUCTION}

Le Comité de Coordination et de Surveillance des Risques Systémiques (CCSRS), chargé par la loi n¹0312 relative aux établissements de crédit et organismes assimilés d'évaluer les risques systémiques pour le secteur financier, a tenu le 23 juin 2017 sa cinquième réunion au siège de Bank Al-Maghrib (BAM) à Rabat.

Lors de cette réunion, le Comité a procédé à l'approbation du rapport sur la stabilité financière relatif à l'exercice 2016, et à l'analyse de la cartographie des risques du système financier, ainsi qu'à l'examen de l'état d'avancement de la feuille de route inter-autorités en matière de stabilité financière au titre de la période 20162018.

L'analyse de la situation du système financier au regard des tendances économiques et financières, observées et attendues, a permis au Comité de dégager les constats suivants :

- Les risques macroéconomiques se sont globalement situés à un niveau modéré. En effet, le risque émanant de la position extérieure demeure limité avec des réserves internationales nettes assurant une couverture de plus de six mois d'importations de biens et services. Sur le plan intérieur, l'économie nationale a été affectée en 2016 par la contreperformance du secteur agricole et l'atonie persistante des activités non agricoles. 
Au niveau des perspectives macroéconomiques, l'évolution reste favorable en lien avec le raffermissement attendu de l'activité économique mondiale et l'accélération prévue en 2017 de la croissance nationale tirée, principalement, par le rebond du secteur agricole et la reprise progressive des activités non agricoles. Parallèlement, le déficit du compte courant et le déficit budgétaire devraient s'atténuer d'ici 2018.

- Le crédit bancaire aux entreprises non-financières a renoué avec la croissance après une contraction constatée en 2015. Le taux des créances en souffrance a, toutefois, enregistré une nouvelle hausse liée à certains secteurs en difficulté.

- Sur la base d'une étude réalisée par Bank Al-Maghrib, portant sur un échantillon de près de 14.000 entreprises non financières privées et publiques, les délais de paiement des créances inter-entreprises se sont de nouveau allongés, en particulier pour les TPME et pour certains secteurs d'activité. La mise en œuvre rapide de la réforme du cadre législatif et réglementaire relatif aux délais de paiement, finalisée au deuxième semestre 2016, devrait contribuer à l'atténuation de cette situation.

- Les bilans bancaires ont été affectés par les effets de la conjoncture économique nationale. Les banques ont ainsi connu une baisse de leurs marges d'intérêt, conjuguée à une nouvelle hausse du risque de crédit sur leurs opérations réalisées au Maroc. Leurs activités bancaires opérées à l'étranger ont, pour leur part, enregistré de bonnes performances. Au global, les banques continuent de disposer d'un bon niveau de capitalisation. Le cadre prudentiel est, par ailleurs, en cours de renforcement à l'effet de consolider la résilience du secteur.

- Le secteur des assurances continue de dégager une marge de solvabilité, en couverture du risque de souscription, largement supérieure au minimum réglementaire. Considérant, néanmoins, le passage futur vers le régime de la solvabilité basée sur les risques, les excédents de marge devraient probablement se réduire de manière significative. Par ailleurs, les plus-values latentes susceptibles d'être dégagées par les portefeuilles d'actifs de ces institutions ont connu une hausse en relation avec la performance du marché boursier.

- La réforme paramétrique du régime des pensions civiles a permis une amélioration de sa situation financière en équilibrant la tarification de ce régime pour les droits futurs de ses affiliés sans, toutefois, résorber ses engagements importants au titre des droits passés.

- S'agissant du marché boursier, sa liquidité s'est inscrite dans une tendance haussière. Elle reste, toutefois, insuffisante en raison essentiellement de la faiblesse du flottant. La volatilité demeure modérée et enregistre une baisse durant les quatre premiers mois de 2017 après deux années de hausses successives. En dépit d'une correction des cours en début d'année, la valorisation de la Bourse de Casablanca ressort à un niveau élevé, tirée par des investisseurs à la recherche de rentabilité dans un contexte de baisse des taux d'intérêts.

- Au niveau du marché de la dette privée, en dépit des difficultés rencontrées par quelques émetteurs, le risque de crédit reste globalement modéré. Le marché a vu l'émission d'obligations subordonnées perpétuelles d'un nouveau type (contingent convertible bonds) présentant un profil de risque différent des émissions habituelles, mais dont le volume global demeure limité. Le Comité a, par ailleurs, fait le point sur la mise en œuvre de la feuille de route relative à la contribution du secteur financier marocain à la promotion du développement durable. Il s'est également penché sur les enjeux de la finance digitale et les risques liés à la cybercriminalité. 


\section{LE SECTEUR BANCAIRE MAROCAIN}

Les banques marocaines sont parmi les plus importantes de toute l'Afrique et dominent le marché marocain. Les sept plus grandes banques du Maroc représentent $90 \%$ des comptes des dépôts totaux du secteur et 82,3\% des actifs de l'industrie, un secteur qui se modernise progressivement et les banques marocaines commencent également à étendre leur portée en Afrique et dans d'autres parties du monde.

Cette expansion n'a pas toujours été totalement sans heurts ni sans risques, mais elle commence à porter ses fruits. Attijariwafa Bank réalise aujourd'hui environ un tiers de ses revenus hors du Maroc, tandis que les revenus internationaux de la Banque Centrale Populaire ont augmenté de 800\% entre 2009 et 2017.

Les banques se développent également dans de nouveaux services. En 2017, BTI Bank a lancé une nouvelle offre de banque islamique, donnant accès à des produits et services conformes à la Charia. Cette offre a connu un succès presque sans réserve, non seulement en stimulant l'inclusion financière des citoyens auparavant non bancarisés et en s'étendant à plus de 100 agences, mais aussi en ouvrant de nouveaux marchés pour la BTI Bank.

\section{3. ÉQUILIBRER L'EXPANSION ET LE RISQUE}

Une telle expansion offre des opportunités de croissance significatives pour les banques marocaines, mais une telle stratégie s'accompagne également d'un risque accru. Cela signifie que les fournisseurs de services financiers ont également besoin du bon logiciel de gestion des risques d'entreprise pour se protéger et atténuer ce risque à mesure qu'ils se développent (figure 1).

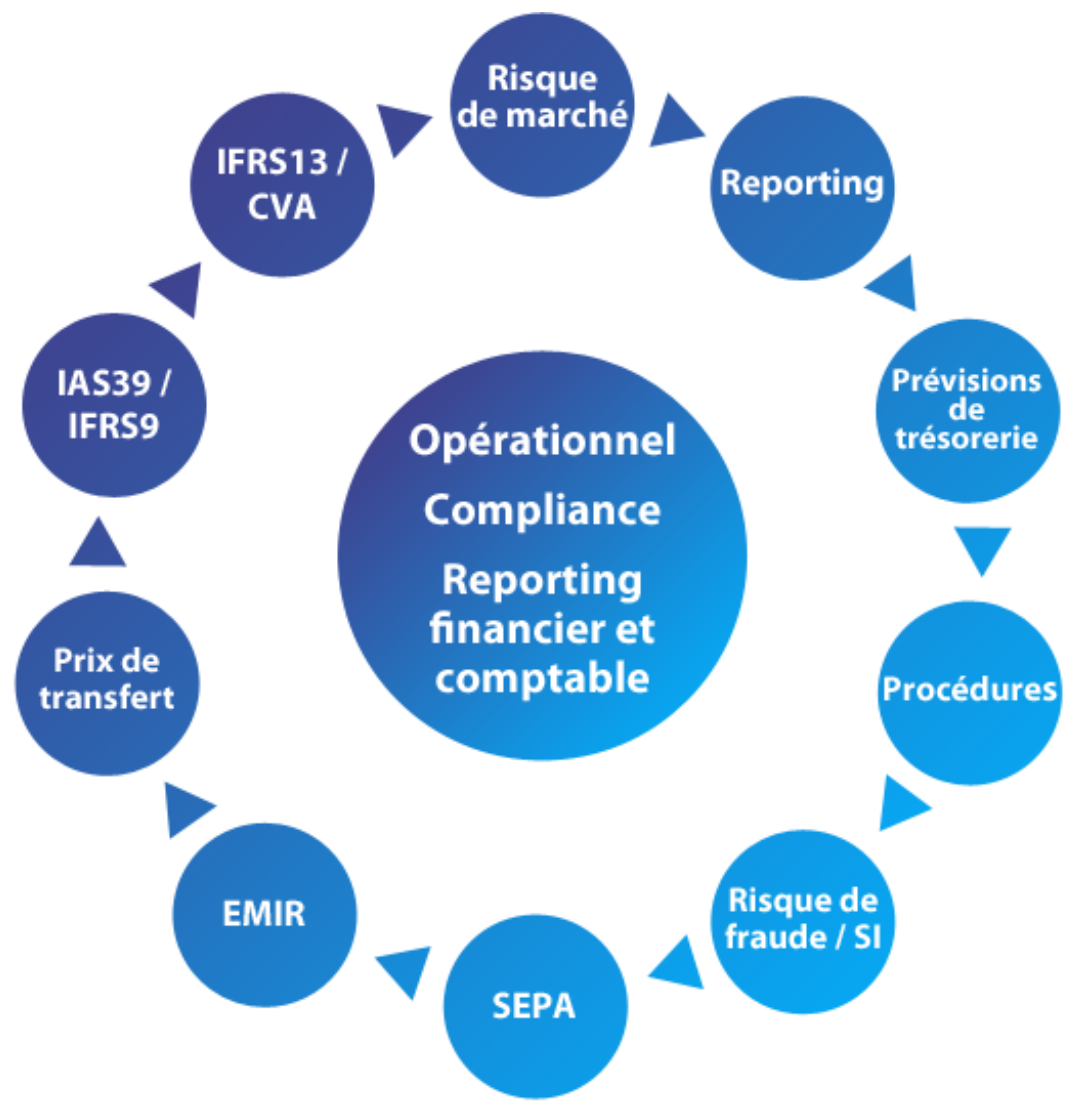

Figure 1 : la collaboration avec les autres fonctions de la Direction Financière et l'intégration dans les processus clés de l'entreprise. 
L'acquisition et l'expansion peuvent exercer une pression sur les réserves d'une banque, alors qu'en Afrique de l'Ouest, où l'expansion est importante, certains pays sont politiquement moins stables que d'autres. En raison de la langue, de la culture et des liens historiques, la France est un marché européen naturel pour l'expansion. Mais l'introduction de GDPR en 2018 a signifié une autre couche de conformité réglementaire à gérer. Toutes les entreprises marocaines de services financiers qui détiennent des données sur les citoyens de l'UE doivent se conformer à la GDPR et les pénalités pour non-conformité peuvent signifier certaines des plus grosses amendes que l'industrie a connues.

Une notation Fitch datant de début 2019, mettait en garde contre les risques qu'elle prétendait faire peser sur les banques marocaines. La banque centrale marocaine a réfuté cette affirmation, déclarant qu'elle avait un contrôle réglementaire efficace et effectif du secteur bancaire, contrairement à ce que Fitch suggérait. Fitch est une agence de notation de crédit internationale respectée, et ses avertissements ne peuvent pas être complètement rejetés.,

\section{LA NECESSITE D'UNE GESTION INTELLIGENTE DES RISQUES}

Il existe sans aucun doute des possibilités d'expansion et de croissance des services financiers marocains, mais même si l'on n'est pas d'accord avec la notation de Fitch, il est clair que le secteur a encore un grand besoin de gérer et d'atténuer les risques plus efficacement à mesure qu'il progresse.

Pour ce faire, il faut disposer de la bonne solution logicielle GRC. Il n'est pas nécessaire que ce soit l'outil de GRC le plus cher du marché, mais il doit être adapté aux besoins et aux objectifs globaux de l'organisation concernée. Oxial a récemment lancé ses nouvelles solutions sGRC - sGRC Express et sGRC Suite - qui portent les fonctionnalités GRC à un niveau supérieur. Dans la figure 2 nous présentons la courbe de Farmer. (Figure 2)

\section{Courbe de Farmer}

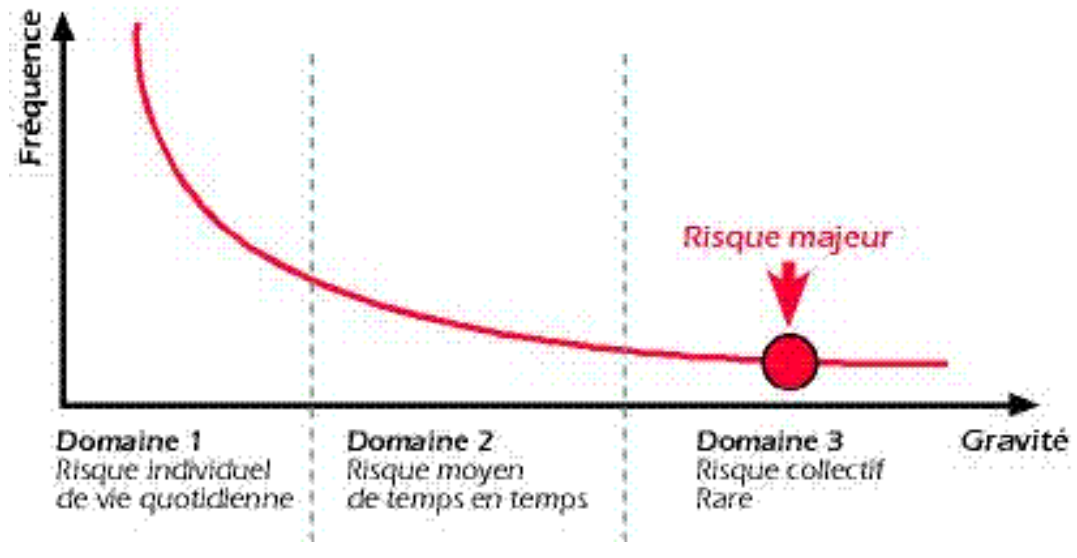

Figure 2 : Courbe de Farmer.

Nous avons intégré la technologie et l'innovation, ainsi que les connaissances, la gouvernance et les processus, la culture et les capacités pour créer une solution qui atténue le risque de manière très efficace et garantit la conformité à toutes les exigences réglementaires. Parce que nos partenaires, nos clients ont accès à certains des consultants les plus compétents en Afrique du Nord, avec une compréhension profonde des services financiers marocains mais aussi du monde entier mesure que les entreprises marocaines de services financiers élargissent leur champ d'action, pénètrent de nouveaux marchés et offrent de nouveaux produits et services, il est logique que les risques augmentent également. Si vous êtes une entreprise marocaine de services financiers 
et que vous souhaitez obtenir de l'aide pour gérer et réduire les risques, veuillez contacter l'un des experts marocains de services financiers ici. Figure 3 présente l'attaque financière représente une arme de choix pour les entreprises se situant dans un contexte de guerre économique. Les vols de documents et le piratage informatique, tellement craints, figurent loin derrière dans le palmarès des attaques contre les entreprises françaises établi par la section financière de la DCRG, le risque financier représentant plus de $35 \%$ des modalités d'agression.

\section{LES RISQUES ET ATTEINTES: REPARTITION PAR GRANDES FAMILLES}

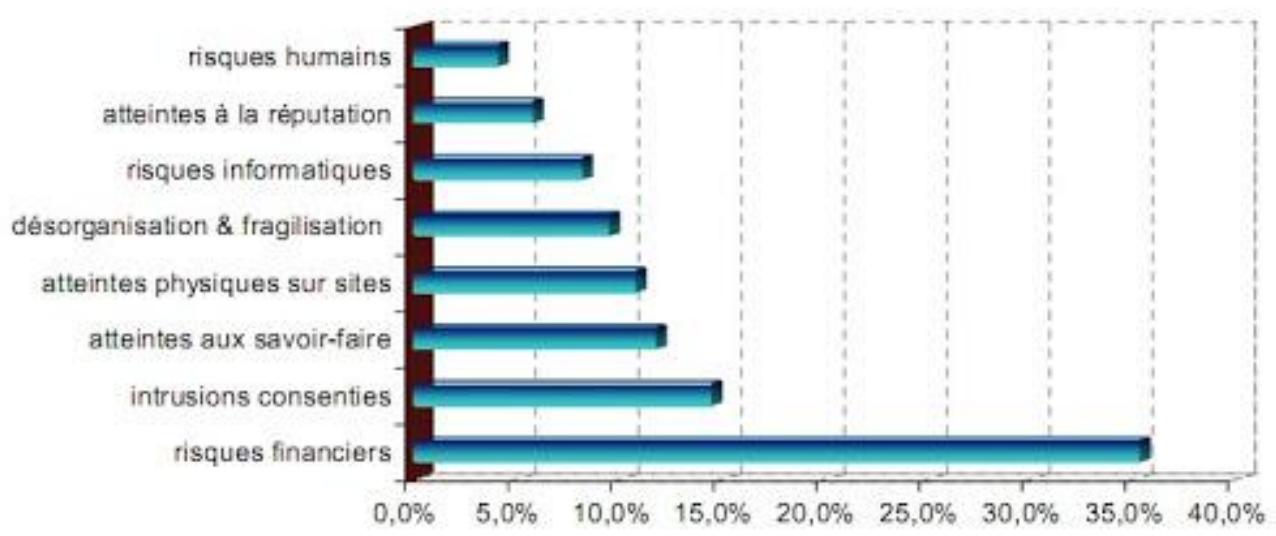

Figure 3 : Les risques financiers identifiés par les ex-RG

\section{CONCLUSION}

La mise en place d'une politique de gestion des risques dans l'entreprise est une compétence professionnelle recherchée. Puisque les risques à couvrir sont intimement liés à la nature de l'activité de l'entreprise, les compétences métiers priment sur les capacités techniques pures. La gestion des risques est un des facteurs-clés de succès de nombreuses entreprises et dépasse aujourd'hui le seul cadre des établissements financiers avec la création de départements dédiés.

\section{REFERENCES}

[1] REBEYROL P. et DECAP A.-S. (2010), « Apparition et développement des fondations dans le paysage juridique français du mécénat », Gestion \& Finances Publiques, n 12, décembre, pp. 918-922.

[2] RUSSO P.-D. (2007), Les CIGALES : notre épargne, levier pour entreprendre autrement, éditions Yves Michel. VANIER $\mathrm{M}$.

[3] GLÉMAIN P. (2018), « Appel public à entreprendre en proximité : une première évaluation du soutien régional au réseau France Active pour l'entreprendre "local". Le cas du FONDES en Pays de la Loire », in Altintas G. et Kustosz I. (coord.), Capacités entrepreneuriales : des organisations aux territoires », Caen, éditions ems-Management \& Société, pp. 161-187.

[4] GLÉMAIN P. et BILLAUDEAU V. (2018), «Finances solidaires et gestion des biens communs : l'expérimentation du Fonds de dotation Angers Mécénat », Marché \& Organisations, n³1, pp. 125-150.

[5] GLÉMAIN P. et LE DEN X. (2008), Évaluation du soutien régional à France Active, Rapport de recherche au Conseil Régional d'Île-de-France, Ramboll Management-Chaire ESS ESSCA EM.

[6] GOMPERS P. A. et LERNER J. (2001), « The Venture Kapital Revolution », Journal of Economic Perspectives, vol. 15, n 2, pp. 145-168. 
[7] GUÉRIN I. et VALLAT D. (2000), « Très petites entreprises et exclusion bancaire en France : les partenariats associationsbanques », Revue d'économie financière, $n^{\circ} 58$, pp. 151-162.

[8] GUILHON B. et MONTCHAUD S. (2003), « Le capital à risque et les jeunes entreprises innovantes : problématique et enjeux », Revue internationale PME, vol. 16, n 3-4, pp. 53-73.

[9] GURTNER E., JAEGER M. et ORY J.-N. (2002), « Le statut de coopérative est-il source dans le secteur bancaire ?», Revue d'économie financière, $\mathrm{n}^{\circ}$ 67, pp. 133-163.

[10] HALL E. T. (1971), La dimension cachée, Seuil.

[11] KPMG (2017), Baromètre du crowdfuunding en France, Financement participatif France publication.

[12] LERNER J. (1998), « Angel Financing and Public Policy: an Overview », Journal of Banking and Finance, vol. 22, pp. 773 783.

[13] LESUR N. (2015), « Les défis posés par le crowdfunding », Revue d'économie financière, n 118 , pp. 103-112.

[14] LÉVY J. et LUSSAULT M. (2003), Dictionnaire de la géographie et de l'espace des sociétés, éditions Belin.

[15] MAHIEU X. (2010), « La gestion déléguée dans les fonds de capital-investissement : relation d'agence etclauses contractuelles des fonds », Revue d'économie financière, $n^{\circ}$ 97, pp. 253-274.

[16] MÉRIC J., MAQUE I. et BRABET J. (2016), International Perspecyives on Crowdfunding. Positive, Normative and Critical Theory, Bingley, Emerald Group.

[17] MORIN S. (2015), Finance solidaire, finance participative : une articulation est-elle possible pour le réseau des

[18] CIGALES de Bretagne ?, Mémoire de Master 2 Administration des entreprises, parcours « création, acquisition, reprise et transmission d'entreprise », Glémain P. (sous la direction de), IGR IAE-Université de Rennes 1, soutenu le 10 avril 2015 à Rennes. 